\title{
Studiengang
}

Armando Schär*

\section{Digital Business Management - eine neue Studienrichtung im Bachelor of Science in Information Science}

https://doi.org/10.1515/iwp-2020-2110

\section{Digital Business Management - ein Spin-off des Bachelor Studiengangs Informationswissenschaft}

Die digitale Transformation führt zu einem eklatanten Fachkräftemangel in der Wirtschaft. Das Bachelor-Studium der Informationswissenschaft bietet eine ideale Ausgangslage, um auf diesen Umstand einzugehen und Fachkräfte für die Digitalwirtschaft auszubilden. Unter Nutzung dieser Synergien wurde im Jahr 2016 die neue Studienrichtung Digital Business Management eingeführt, mit der eine erweiterte Zielgruppe Studierender angesprochen werden konnte. Der Aufbau des Studiums wurde, ausgehend von informationswissenschaftlichen Kompetenzen, auf spezifische Bedürfnisse digitaler Projekte in der Schweizer Digitalwirtschaft ausgerichtet. So ersetzten Module rund um E-Business Management, Innovationsmethodik, Projektmanagement und Entrepreneurship vertiefende Inhalte aus dem informationswissenschaftlichen Studium. Rund 200 Studierende werden derzeit in dem Studienangebot ausgebildet. In den ersten vier Jahren des Programmes wurden über 60 Studierendenprojekte mit Unternehmenspartnern umgesetzt. Die ersten Absolventinnen und Absolventen des Programms des Abschlussjahrs 2019 wurden sehr gut vom Arbeitsmarkt angenommen.

\section{Weiterentwicklung 2020}

Zum Start des Herbstsemesters 2020 wurde im Zusammenhang mit der Selbstständigkeit der Fachhochschule Grau-

*Kontaktperson: Prof. Armando Schär, Fachhochschule Graubünden, Schweizerisches Institut für Informationswissenschaft, Pulvermühlestrasse 57, 7000 Chur, Schweiz, E-Mail: armando.schaer@fhgr.ch bünden eine weitreichende Curriculumsreform umgesetzt. Der bisher recht starr vorgegebene Lernpfad wurde flexibilisiert. Von bisher vier Wahlpflichtmodulen belegen die Studierenden nun dreizehn Wahlpflichtmodule in ihrem Studium. Dabei können sie aus drei neuen Schwerpunkten auswählen. Dieser Ausbau des Studienganges erlaubt es, besser auf die Bedürfnisse der digitalen Wirtschaft einzugehen und den Studierenden ein individuelles Studium anzubieten. Auch weiterhin sind mit mindestens 66 ECTS viele Überschneidungen mit dem Bachelor Studium Informationswissenschaft vorhanden.

\section{Neue Schwerpunkte}

Der Schwerpunkt User Experience befasst sich mit den Bedürfnissen von Nutzenden, deren Erhebung und deren Umsetzung in digitalen Lösungen. Dabei werden Konzepte und Prototypen erstellt und in Usability-Evaluationen auf ihre Nutzerfreundlichkeit hin getestet. In den vielseitigen Modulen aus dem Schwerpunkt Digital Innovation widmen sich Studierende dem Umgang von Unternehmen mit der Digitalisierung. Sie beschäftigen sich unter anderem damit, Projekte agil zu leiten, Online-Marketingmaßnahmen gezielt einzusetzen sowie Innovationsmanagement-Methoden wie Design Thinking anzuwenden. In einem sich so schnell entwickelnden Umfeld wie der Digitalisierung müssen Fachkräfte up-to-date bleiben und sich deshalb im Studium technische Mitsprachekompetenz erwerben. Im Schwerpunkt Information Technology erlernen die Studierenden deshalb IT-Grundlagen und entwickeln ein grundlegendes Verständnis für den Software-Entwicklungsprozess, um bei digitalen Projekten die Führung von Fachkräften zu übernehmen.

Der Studiengang befähigt, aktuelle Trends und digitale Geschäftsmodelle zu analysieren und zu bewerten, innovative E-Business Lösungen zu konzipieren und deren Umsetzung zu begleiten sowie digitale Projekte zu leiten und zu koordinieren. 


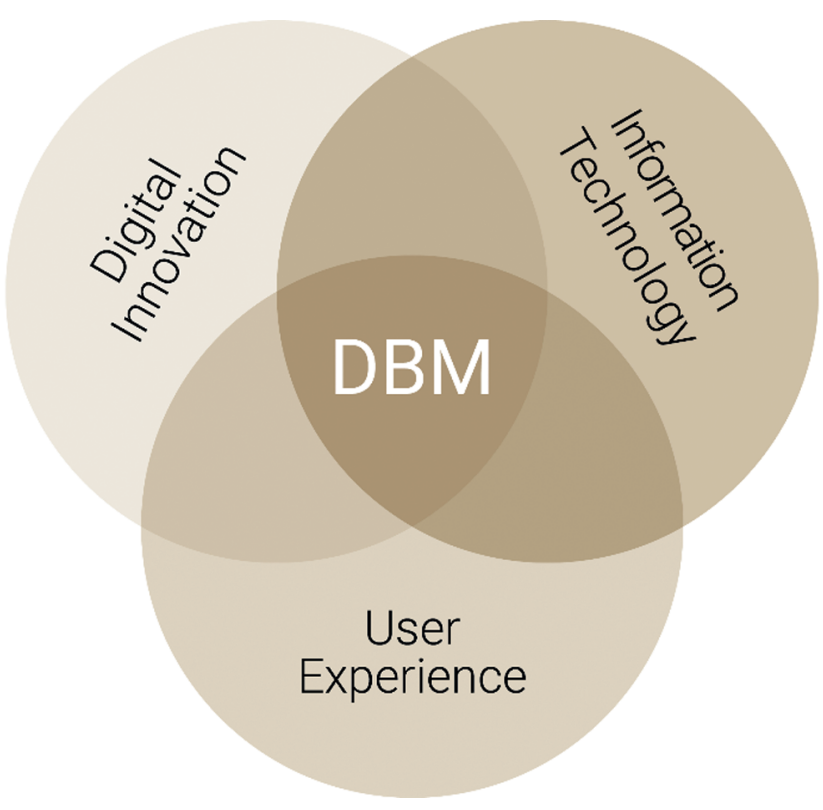

Abbildung 1: Studienschwerpunkte Digital Business Management.

\section{Kompetenzziele und Berufsbilder}

Das Studienangebot orientiert sich an den in der Wirtschaft geforderten Berufsprofilen rund um die Konzeption, Beratung und Leitung digitaler Projekte. Der rasante Fortschritt der Digitalisierung verlangt Unternehmen große Anstrengungen ab. So müssen alle Kunden-Interaktionen auf die Ausweitung auf digitale Kanäle überprüft werden. Daraus resultierende Digitalisierungsprojekte führen $\mathrm{zu}$ weitreichenden Prozessanpassungen. Dies bedingt nicht nur spezifisch ausgebildete Konzepterinnen und Konzepter, sondern bei der Projektleitung auch neue Leitungsund Koordinationsmethoden, um digitale Projekte in einem dynamischen Umfeld erfolgreich durchführen $\mathrm{zu}$ können. Insbesondere die große Überschneidung von Anforderungen an das Nutzererlebnis, die Nutzung neuster Informationstechnologien und Konzeptions- und Umsetzungsmethoden erfordern die spezifische Ausbildung dafür geeigneter Berufsprofile:

\section{Einsatzbereiche in der Praxis}

Digitale Geschäftsprozesse und junge Unternehmen mit neuartigen, disruptiven Geschäftsmodellen verändern zurzeit die Wirtschaft auf rasante Art und Weise. Unternehmen investieren in die Digitalisierung und sind auf spezifisch ausgebildete Fachkräfte angewiesen, die mit der Entwicklung Schritt halten und Unternehmen in das digitale Zeitalter begleiten können. Während vor wenigen Jahren bei den Unternehmen vor allem die Kostenreduktion durch die Schaffung von Kundenportalen im Fokus stand („,hier können Sie Ihre Fragen online stellen, bitte rufen Sie uns nicht mehr über die Hotline an..."), wird heute versucht, neben Kostenersparnissen, die Bedürfnisse der Kundinnen und Kunden möglichst einfach, orts- und geräteunabhängig zu erfüllen. Eines der vielen Beispiele sind die Bestrebungen der Schweizerischen Bundesbahnen bis 2023 die Hälfte der Tickets über die Smartphone App zu vertreiben. Neuartige und innovative Dienste erleichtern Kundinnen und Kunden den Alltag weit über die schon länger etablierten Online Shops hinaus. Beispiele, wie die Schweizer Bezahlapplikation Twint (ein Joint-Venture diverser Schweizer Banken) oder der Abschluss von Online Versicherungen und Hypotheken, gibt es bereits viele.

\section{Ausblick}

Mit dieser Weiterentwicklung des Studiengangs Informationswissenschaft werden zentrale Elemente eines informationswissenschaftlichen Studiums auf Unternehmensbedürfnisse der digitalen Transformation angewandt. Die ersten Absolventinnen und Absolventen starteten ihre Karriere im Herbst 2019. Ihre Rückmeldungen werden Einfluss auf die nächste Curriculumsreform haben. Durch die stärkere Gewichtung der Wahlpflichtmodule können künftig einfacher Anpassungen am Curriculum vorgenommen werden, indem das Angebot an Wahlpflichtmodulen gezielt gesteuert wird. Um mit der schnellen Entwicklung der digital getriebenen Wirtschaft mitzuhalten und die passenden Kompetenzen auszubilden, wird mit einem agilen Studiengang auf die agile Wirtschaft reagiert.

Zum Studienprogramm: www.fhgr.ch/dbm

Deskriptoren: Wirtschaft, Digitalisierung, Management, Studium, Digital Business Management, Schweiz

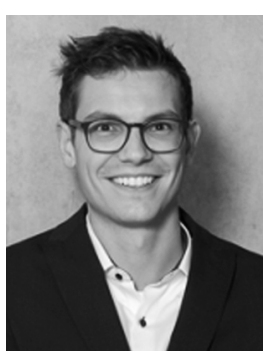

\author{
Prof. Armando Schär \\ Fachhochschule Graubünden \\ Schweizerisches Institut für \\ Informationswissenschaft \\ Pulvermühlestrasse 57 \\ 7000 Chur \\ Schweiz \\ armando.schaer@fhgr.ch
}

Prof. Armando Schär ist studierter Wirtschaftswissenschaftler (Informations-, Medien- und Technologiemanagement, Universität St.

Gallen) und diplomierter Wirtschaftspädagoge. Als Studienleiter verantwortet er das Bachelor Studienangebot Digital Business Management an der Fachhochschule Graubünden. 